\title{
Le district industriel : Une revue de littérature
}

\author{
Nada El Khatir, Doctorante en sciences \\ économiques et gestión
}

Laboratoire de recherche en intelligence stratégique

Faculté des Sciences Juridiques, Economiques et Sociales de Mohammedia

Université Hassan II de Casablanca, Maroc

Doi:10.19044/esj.2020.v16n19p365 URL:http://dx.doi.org/10.19044/esj.2020.v16n19p365

\section{Résumé}

Les formes d'organisation industrielle ont évolué à travers l'histoire pour accompagner les changements des contextes économiques et sociaux. Le monde des affaires en connaît de nos jours bien des variantes allant de l'écosystème d'affaires à la technopole en passant par le milieu innovateur. Afin de mieux saisir le fonctionnement de ces nouvelles formes d'agglomération, il est important de revenir sur celle qui en a constitué l'ébauche première : le district industriel. Ce modèle organisationnel reposant principalement sur des petites firmes dont la communauté locale constitue la principale force motrice, a su se montrer efficace là où des modèles reconnus comme «solides » notamment la production de masse ont atteint leurs limites. Cette revue de littérature narrative se base sur une lecture analytique approfondie des publications scientifiques ayant le plus marqué la construction du concept de district industriel, à savoir l'héritage de Marshall et les contributions des économistes italiens. Il est ainsi question de définir le district industriel et de le replacer dans son contexte historique dans un premier temps, puis d'en étudier les spécificités les plus discutées dans la littérature, afin d'en présenter une introduction scientifique complète.

Mots-clés: District industriel agglomération, petites firmes, communauté locale 


\title{
The Industrial District: A Literature Review
}

\author{
Nada El Khatir, Doctorante en sciences \\ économiques et gestión
}

Laboratoire de recherche en intelligence stratégique

Faculté des Sciences Juridiques, Economiques et Sociales de Mohammedia

Université Hassan II de Casablanca, Maroc

\begin{abstract}
Through history, several organizational forms in the industrial field have been developed to match the economic and social context. Today, many are to be found in the business field such as business ecosystems, technopoles and innovative milieux. To study these recent industrial configurations, it is of crucial importance to take the time to go back to the very first one of them: the industrial district. Be it in times of crises, or well being, these agglomerations of little firms which mainly employ the local community proved its efficiency when other forms failed. The purpose of this paper is to present the industrial district and its main features, starting with Marshall's legacy and building the concept's framework progressively on the contributions of Italian economists. This narrative literature review does not claim to be exhaustive. It is based on the analysis of scientific publications recognized as major milestones of the concept's development. These publications have been carefully studied and sorted to define the industrial district and outline its underpinnings.
\end{abstract}

Keywords: Industrial district, agglomeration, little firms, local community

\section{Introduction}

Alors que cela relève aujourd'hui de l'évidence, les chercheurs en sciences économiques ont longtemps été rigides face à toute tentative d'entremêler le social et l'économique. En effet, le consensus théorique classique consistait à étudier les modèles d'équilibre économiques " pour des circonstances sociales données ", excluant ainsi du champ de l'analyse tout paramètre de nature sociale. C'est à partir des années 1970 que l'aspect social commencera à être intégré dans l'analyse des phénomènes économiques grâce à l'introduction de nouveautés théoriques substantielles telles que la théorie des jeux qui suggère la modélisation de l'interaction rationnelle entre agents.

Ces dernières décennies, la littérature économique s'est enrichie de nouveaux éléments de la dynamique des relations sociales. Des notions 
psychologiques telles que la confiance, l'altruisme, et la réciprocité sont devenues essentielles dans de nombreuses recherches contemporaines aussi bien théoriques qu'empiriques. C'est à ce renouveau de la pensée économique que l'on doit le regain d'intérêt pour l'œuvre d'Alfred Marshall, un grand économiste qui considérait l'évolution de la science économique comme une partie de la grande mosaïque qu'est la science sociale à travers l'histoire.

Aujourd'hui, il est difficile de mener un travail de recherche sur l'organisation industrielle, sans se référer aux apports de Marshall en la matière. Ce dernier fut le premier à s'intéresser aux agglomérations de petites firmes du Lancashire et du Yorkshireet et à affirmer que vie sociale et économique sont deux facettes d'un même processus de développement. Il défendait la nécessité d'orienter l'intelligence de l'économiste vers les problèmes qui touchaient des sujets en chair et en os, afin d'accélérer le rythme de développement de la connaissance sociale. Il est donc vrai que les idées de Marshall avait plutôt une portée philosophique et ne fournissaient pas les instruments nécessaires à une analyse économique, elles étaient toutefois avant-gardistes dans un contexte d'orthodoxie économique.

Cet article propose de faire le tour de la littérature académique ayant traité les aspects les plus importants du concept de «district industriel » tel que présenté d'abord par Marshall puis analysé par d'autres chercheurs notamment Becattini. Cette revue de littérature narrative a été développé suivant une méthodologie de sélection des sources bibliographiques les plus pertinentes, à savoir les travaux des auteurs qui ont façonné le concept de district industriel depuis son apparition et qui ont fortement impacté sa perception. Dans un premier temps, la recherche bibliographique s'est focalisée sur les travaux définissant le concept et expliquant sa genèse, ensuite elle s'est orientée vers ceux couvrant les principales caractéristiques d'un district industriel dans une perspective qui, sans prétendre à l'exhaustivité, tente de fournir au lecteur un aperçu de l'essentiel des connaissances produites dans ce sens.

\section{Qu'est ce que le district industriel ?}

La notion de district industriel est aujourd'hui considérée comme une référence indispensable en matière d'organisation industrielle. C'est à l'économiste anglais Alfred Marshall qu'on attribue la paternité de ce concept figurant initialement dans son ouvrage «Principles of economics » publié pour la première fois en 1890. Dans ce livre, Marshall ne s'est pas penché sur la notion de district de manière directe, mais plutôt en marge d'autres thématiques se rapportant principalement aux dynamiques de la production. Il avança ainsi, en désaccord avec la plupart de ses contemporains, que les avantages de la division du travail obtenus dans les grandes usines pouvaient également être observés dans les petites, voire très petites entreprises. Il suffit 
pour cela que chacune de ces firmes s'occupe d'une étape du processus de production d'un même bien qui sera produit en quantité conséquente, mais aussi et surtout qu'elles soient localisées dans un même district (Marshall, 2009).

Bien après, le besoin de produire un cadre théorique pouvant contenir la réalité de l'économie italienne fut à l'origine du regain d'intérêt pour le travail de Marshall. Ce fut l'économiste italien G. Becattini qui retraça en premier, au début des années 1980, le contour du district industriel tel qu'on le connait aujourd'hui, grâce à une analyse approfondie de l'œuvre du père de la Cambridge School. Le contexte économique de l'époque est marqué par la naissance de ce qu'on a appelé " la troisième Italie », venue compléter le duo formé par le Nord-est développé (Milan, Turin, Gênes) et le Sud sous développé. Cette Italie nouvelle qui prenait la forme de systèmes productifs spécialisés dans des activités variées, notamment le textile en Toscane, la chaussure à Marches et les meubles en bois en Vénétie, avait, aux yeux des économistes italiens de l'époque, besoin de s'inscrire dans un référentiel académique.

Becattini a ainsi défini le district industriel comme une entité socioterritoriale caractérisée par la coexistence d'une communauté de personnes et d'une population de firmes dans une zone géographique délimitée (Becattini, 1990). La grande variété d'agglomérations existantes sur le terrain ne facilitait pas la tâche aux chercheurs qui se sont essayés à la définition du district. Pyke et Sengenberger l'ont notamment présenté dans l'ouvrage « industrial districts and inter-firmco-operation in Italy » comme un système productif défini géographiquement, marqué par la présence de nombreuses entreprises impliquées dans différentes étapes et de différentes manières dans la production d'un bien homogène (Pyke et Sengenberger,1990).

Ainsi, tout lecteur attentif de la littérature sur les districts industriels ne peut manquer de remarquer la mention simultanée qui revient fréquemment du couple «communauté de personnes- firmes ». Une mention qui rappelle l'imbrication entre l'économique et le social telle que présentée par Weber dans son ouvrage «Economie et Société » à travers les concepts de communalisation et de sociation (Weber, 1971). La communalisation renvoie au lien social fondé sur le sentiment d'appartenance, et la sociation au pacte rationnel conclu sur la base d'intérêts communs. Cette imbrication est également présente dans les écrits de Marshall, plus précisément «Les principes d'économie politique », ouvrage qui met la lumière sur cet aspect social du district en posant sur la théorie de la localisation un regard différent. En effet, là où les économistes cherchaient à expliquer le phénomène d'agglomération par la mise en avant de variables exogènes, Marshall considérait celles-ci comme étant des raisons accidentelles ayant aboutit à la 
localisation. Ce qui lui parut plus intéressant à analyser c'est ce que la localisation produit intrinsèquement comme avantages.

Marshall a ainsi scindé ces avantages en deux catégories : la première illustre la fameuse « atmosphère industrielle »provenant du rassemblement du capital humain qui fait que « les secrets de l'industrie cessent d'être des secrets, ils sont pour ainsi dire dans l'air » (Marshall, 2009). Dans son livre l'auteur souligne l'importance que revêt cette centralisation de ressources humaines dans l'échange et la transmission du savoir et des compétences, encore mieux, dans l'innovation et l'apparition d'idées nouvelles. La seconde catégorie d'avantages est celle des économies externes. Celles-ci naissent lorsqu'une firme spécialisée dans une étape spécifique d'un processus de production arrive à collaborer avec plusieurs industries similaires dans le cadre d'une bonne stratégie de réseautage, ce qui justifie son investissement dans des moyens de production performants et efficaces qui permettront d'assurer sa rentabilité.

\section{La genèse du district industriel et le déclin du fordisme}

Il est généralement admis en milieu scientifique que l'apparition du district industriel est intimement liée au déclin du fordisme. En effet, à partir du milieu des années 1970, le capitalisme s'est retrouvé en croisée de chemins. Le monde vivait une période de transition rompant avec ce qu'a qu'il a été après la deuxième guerre mondiale. : un monde prédominé par le régime de production de masse. De nouvelles notions ont été conçues pour décrire la nouvelle ère qui s'annonçait et que l'on qualifiait de post-industrielle, postfordiste, ou encore post-moderne. Afin de faire le lien entre le déclin du fordisme et la naissance du district industriel, il est important de revenir sur les trois grandes théories qui ont marqué le débat post-fordiste, à savoir la théorie de la régulation, la théorie néo-schumpetérienne et la théorie de la spécialisation flexible. Ces théories ont toutes tenté de jeter les jalons d'une base conceptuelle expliquant pourquoi la production de masse devait céder sa place à une nouvelle forme d'organisation industrielle pouvant soutenir un long cycle économique à venir (Amin, 1994).

\section{La théorie de la régulation}

Cette théorie tente d'expliquer le paradoxe inhérent à la nature même du capitalisme. Celui-ci réside dans sa tendance à l'instabilité, à la transformation à travers des périodes de crise et à sa capacité à se stabiliser autour de normes, de lois et d'institutions permettant de garantir un long cycle de stabilité économique. Pour les adeptes de cette théorie, une phase donnée ne peut être vue comme la simple résultante de lois économiques conjuguées, car elle est avant tout le fruit de pratiques et de lois sociales enracinées (Jessop, 1992 b). Il est donc essentiel d'accorder autant d'importance à l'évolution 
historique qu'aux lois économiques; l'objectif étant d'identifier les mécanismes ayant régi l'ancienne époque avec leurs contradictions pour en dégager de nouvelles possibilités de croissance.

Ce travail d'analyse est mené dans le cadre de la théorie de régulation à travers la mise en place d'un ensemble de concepts dont deux sont les plus importants, à savoir le régime d'accumulation et le mode de régulation. Le régime d'accumulation se définit comme «un ensemble de régularités assurant une progression générale et relativement cohérente à l'accumulation du capital, c'est-à-dire permettant de résorber ou d'étaler dans le temps les distorsions et déséquilibres qui naissent en permanence du processus luimême » (Boyer, 1986, p 46). Autrement dit, il s'agit des normes régissant l'organisation de la productivité, du travail, les échanges entre les branches de l'économie, la répartition des revenus, etc. La question qui se pose ici est : comment régénérer ces régularités si importantes à l'accumulation du capital ? La réponse est apportée par le deuxième concept de "mode de régulation » qui se présente comme l'ensemble des institutions, des pratiques sociales et culturelles assurant la reproduction du capitalisme. Il se décline ainsi en des règles formelles mais aussi informelles s'appliquant aux relations sociales principales dans l'économie.

Nielsen (1991) mentionne trois concepts à rajouter à l'approche de la régulation pour un meilleur diagnostic du profil capitaliste, à savoir «le paradigme industriel dominant» qui a trait au modèle industriel, les technologies qu'il utilise, la nature du management et des relations industrielles, la division des tâches, etc. Le deuxième concept est celui du «mode de développement » avancé par Lipietz qui identifie la trajectoire de développement d'une économie à travers le régime d'accumulation, le mode de régulation et le paradigme industriel dominant. Enfin, le dernier concept est celui du « paradigme sociétal » introduit par Jessop et Lipietz et qui se focalise sur les cohésions sociales et politiques menant à la stabilisation du développement. C'est ainsi qu'à travers ces cinq concepts, l'école de la régulation a pu diviser l'évolution capitaliste en périodes et interpréter systématiquement les situations de crise comme des périodes où les fondements de ces cinq concepts se sont effondrés.

Le fordisme a connu son âge de gloire durant les années 1950 et 1960 marquées par la prédominance de la production de masse qu'Henry Ford préconisait dans ses usines de fabrication de voitures aux Etats-Unis. Si l'on s'applique à analyser le fordisme avec l'œil d'un économiste de l'école de régulation, on le considérerait comme un régime d'accumulation intensive reposant sur la dynamique de la production de masse, l'intensification du travail, la minutieuse division du travail en petites tâches, et la mécanisation de la chaine de production. En effet, ce régime d'accumulation est une sorte de cercle de croissance qui commence par la production de masse. Celle ci 
permet d'augmenter la productivité grâce aux économies d'échelles. Les salaires étant liés à la productivité, la demande de masse voit son niveau fléchir avec l'augmentation des salaires. Par conséquent, les profits sont de plus en plus importants permettant de réaliser de nouveaux investissements qui vont encore augmenter la capacité de production et permettre à ce cycle de recommencer. S'agissant du mode de régulation du fordisme, il a été décrit comme monopolistique, séparant la propriété de la gestion dans les grandes firmes, et indexant les salaires sur la productivité.

Le régime d'accumulation et le mode de régulation de cette ère de production de masse a montré plusieurs limites, en l'occurrence la résistance des ouvriers face au mode organisationnel, la difficulté à mettre en place des lignes de production de plus en plus longues et rigides, la globalisation des flux économiques mettant la gestion des économies locales en difficulté et le changement des habitudes de consommation ayant tendance à s'éloigner des biens standardisés. Ainsi, le district a pu prouver sa crédibilité comme nouvelle forme d'organisation industrielle reposant sur le paradigme de spécialisation flexible et de désintégration verticale, et mettant en valeur la division sociale du travail et la coordination à travers la proximité géographique, des aspects qui seront développés ultérieurement dans cette revue.

Figure $\mathbf{n}^{\circ} \mathbf{1}$ : Le régime d'accumulation du fordisme selon la théorie de régulation

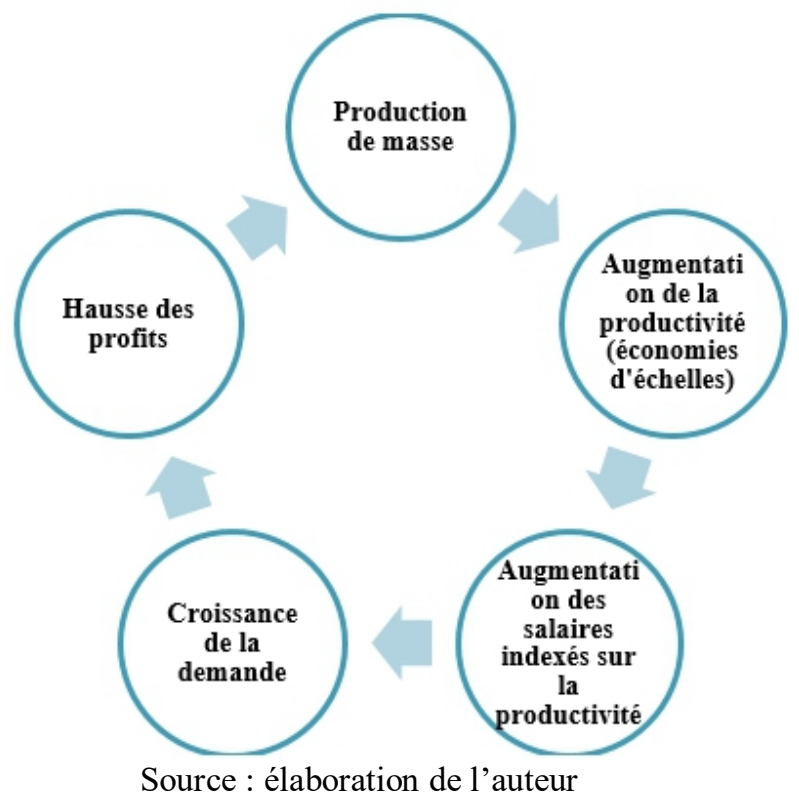

\section{La théorie néo-schumpetérienne}

La théorie néo-Schumpetérienne s'accorde avec la théorie de la régulation sur un nombre de points, notamment l'évolution cyclique du 
capitalisme, l'existence de longs cycles économiques stables et la dynamique du fordisme. Les deux théories proposent également des appellations ayant des significations rapprochées telles que « le paradigme techno-économique » qui s'apparente au «régime d'accumulation», et «la structure socioinstitutionnelle » qui avoisine le concept de «mode de régulation ». Il existe toutefois un point de divergence entre les deux théories consistant en l'importance accordée, dans la théorie néo-schumpetérienne, à la technologie comme facteur initiateur de longs cycles économiques. En effet, la théorie néoschumpetérienne est associée aux travaux de Freeman et Perez et leurs collègues durant les années 1980. Ces derniers se sont basés sur les travaux de Kondratiev qui a affirmé pendant les années 1920 l'existence de vagues longues de cinquante ans, alternant boom et récession économiques dans l'évolution du capitalisme (Freeman et Perez, 1988). Cette réflexion a ensuite été développée par Schumpeter pendant les années 1930. Il y introduit l'idée selon laquelle les entrepreneurs innovants ont un rôle décisif dans la création de nouveaux paradigmes techniques et technologiques qui sont à l'origine de nouveaux cycles de croissance.

Une transition réussie est souvent liée à l'apparition d'un changement brusque dans la productivité industrielle, ce que Freeman et Perez ont appelé " Quantum leaps ». Ce changement doit être diffusé dans l'économie en question et être accompagné d'une émergence parallèle de normes et de lois dans la structure socio-institutionnelle permettant cette diffusion. Une fois ces deux conditions réunies, un nouveau cycle économique peut commencer avec un paradigme techno-économique bien spécifique. Il est à souligner que dans la théorie néo-schumpetérienne, le mot innovation ne se limite pas à de nouveaux produits ou processus industriels, mais il inclue également les nouvelles formes d'organisation de travail, les nouvelles technologies de communication, de transport, les nouvelles formes de management,etc.

Après cette brève présentation de la théorie, il convient de revenir à l'analyse de l'ère de production de masse en l'utilisant comme cadre de référence. Le fordisme serait ainsi le quatrième cycle de Kondratiev (les trois cycles précédents s'étalant de 1780 à 1930) soutenu par un paradigme technoéconomique basé sur les technologies électromécaniques. Cette vague économique est marquée par l'utilisation des produits pétrochimiques comme sources d'énergie peu couteuses, la standardisation de la production, la massification, les économies d'échelles, la concurrence oligopolistique, la consommation massive de biens bon marché et l'organisation verticale du processus de production dans les grandes firmes. Pour ce qui est de l'aspect socio-institutionnel, la théorie néo-schumpetérienne place au cœur de son 
analyse les politiques publiques et leur impact direct sur le fragile équilibre économique.

La crise du quatrième cycle de Kondratiev est expliquée principalement par le décalage entre le paradigme techno-économique censé relancer la croissance, et la structure socio-institutionnelle en place à l'époque. Selon Freeman et Perez, cette structure ne suivait pas le rythme de changement imposé par le nouveau paradigme et empêchait donc sa diffusion à travers l'économie. Ce problème a été considéré comme symptomatique de l'incapacité des politiques publiques à assurer la coordination nécessaire entre les milieux économiques, sociaux et institutionnels, mais il a également été lié à l'enracinement des normes socio culturelles sur lequel il est difficile d'agir dans le laps de temps demandé.

\section{La théorie de la spécialisation flexible}

La théorie de la spécialisation flexible est attribuée aux sociologues américains Charles Sabel, Michael Piore et Johnattan Zeitlen, puis plus récemment à Paul Hirst (Piore etSabel ,1984; Sabel et Zeitlen, 1985 ; Hirst et Zeitlen, 1989,1991). Selon Amin (1994), cette théorie est conceptuellement plus simple que les deux précédentes, dans le sens où elle se focalise sur la production industrielle en en distinguant deux formes. D'une part, la production de masse concentrée sur un produit standardisé, la mécanisation de la chaine de production et une force de travail sans compétences notables. D'autre part, la spécialisation flexible dite aussi « craft production », reposant principalement sur les compétences pointues des employées qui fabriquent des biens personnalisés (Piore et Sabel, 1984). C'est ce dernier mode de production qu'est la spécialisation flexible qu'on retrouve dans le district industriel. Amin explique que ces deux paradigmes industriels ont coexisté depuis le $19^{\text {ème }}$ siècle sans que l'un ne surpasse forcément l'autre. Toutefois, à travers l'histoire, il est arrivé que l'un ait été adopté au détriment de l'autre suite à des circonstances historiques particulières, des choix politiques et des orientations des acteurs principaux (gouvernements, firmes, unions,etc) qui tendent à favoriser un mode de production à l'autre. Les moments de l'histoire où l'un des deux paradigmes manque de structures pouvant le soutenir, alors que l'autre prend le dessus sur le terrain économique, ne sont pas facilement identifiables : s'agit-il des moments suivant une crise de l'un des deux paradigmes ? Ou de conséquences de décisions institutionnelles forcées ? Estil question de l'émergence de nouveaux moyens et de nouvelles opportunités ? Les explications qui sont données n’ont pas de bases théoriques, mais se rapportent plutôt aux événements particuliers ayant marqué ces moments précis de l'histoire.

Piore et Sabel ont ainsi identifié deux « ères industrielles », ce qu'ils appellent « industrial divide ». La première au début du siècle dernier marquée 
par l'émergence de la production de masse qui, à travers son recours aux technologies, a réduit l'intérêt pour la production artisanale dans de nombreuses régions d'Europe pendant les années 1920 et 1930. Les forces économiques adoptent ainsi ce nouveau mode de production et les politiques publiques s'y adaptent. Vint ensuite l'ère de la stagnation à partir des années 1970 marquée par la crise du fordisme, symbole de la production de masse, et offrant ainsi une nouvelle opportunité à la spécialisation flexible, d'où l'émergence des districts industriels comme modèle réussi de production.

Tableau $\mathbf{n}^{\circ} \mathbf{1}$ : Les trois théories explicatives du déclin du fordisme

\begin{tabular}{|c|c|c|c|}
\hline & \multicolumn{2}{|c|}{ Caractéristiques de l'ère du Fordisme } & $\begin{array}{l}\text { Explication du déclin } \\
\text { du Fordisme }\end{array}$ \\
\hline $\begin{array}{l}\text { La théorie de la } \\
\text { régulation }\end{array}$ & $\begin{aligned} & \text { Régime d'accumulation : } \\
& \text { - } \text { Production de } \\
& \text { masse } \\
& \text { - } \text { Intensification de } \\
& \text { travail } \\
& \text { - } \text { Division des taches } \\
& \text { - } \text { Mécanisation de la } \\
& \text { chaine de } \\
& \text { production }\end{aligned}$ & $\begin{array}{ll}\text { Mode de régulation : } \\
\text { - } & \text { Indexation } \\
& \text { des salaires } \\
& \text { sur la } \\
& \text { productivité } \\
\text { - } & \text { Séparation } \\
& \text { entre gestion } \\
& \text { et propriété } \\
\text { - } & \text { Monopole }\end{array}$ & $\begin{array}{ll}\text { - } & \text { Résistance des } \\
\text { ouvriers } \\
\text { - Changement } \\
\text { des habitudes } \\
\text { de } \\
\text { consommation } \\
\text { - Difficulté } \\
\text { rencontrées par } \\
\text { les économies } \\
\text { locales face à la } \\
\text { mondialisation }\end{array}$ \\
\hline $\begin{array}{l}\text { La théorie Néo } \\
\text { Schumpétérienne }\end{array}$ & $\begin{array}{l}\text { Paradigme techno- } \\
\text { - } \frac{\text { économique : }}{\text { Standardisation de }} \\
\text { la production } \\
\text { - Organisation } \\
\text { verticale du } \\
\text { processus de } \\
\text { production } \\
\text { - Economies } \\
\text { d'échelles } \\
\text { - Consommation } \\
\text { massive des biens } \\
\text { bon marché } \\
\text { - Sources d'énergies } \\
\text { peu couteuses: } \\
\text { produits } \\
\text { pétrochimiques } \\
\end{array}$ & $\begin{array}{l}\frac{\text { Structure socio- }}{\text { institutionnelle : }} \\
\text { Les politiques } \\
\text { publiques en matière } \\
\text { d'économie } \\
\text { d'éducation, etc jouent } \\
\text { un rôle important dans } \\
\text { l'entretien du cercle } \\
\text { vertueux reliant } \\
\text { l'emploi, la } \\
\text { productivité, et la } \\
\text { croissance. }\end{array}$ & $\begin{array}{l}\text { Décalage entre le } \\
\text { rythme d'évolution du } \\
\text { paradigme techno- } \\
\text { économique, et la } \\
\text { structure socio- } \\
\text { institutionnelle de } \\
\text { l'époque. }\end{array}$ \\
\hline $\begin{array}{l}\text { La théorie de la } \\
\text { spécialisation } \\
\text { flexible }\end{array}$ & $\begin{array}{l}\text { - } \quad \text { Main d'œuvre peu } \\
\text { - } \quad \text { Produits standardisé } \\
\text { - } \quad \text { Production massive } \\
\text { - } \quad \text { Chaines de producti }\end{array}$ & lalifiée & $\begin{array}{l}\text { La prédominance d'un } \\
\text { mode de production } \\
\text { particulier est le résultat } \\
\text { de faits historiques, de } \\
\text { choix et d'orientations } \\
\text { des acteurs politiques et } \\
\text { économiques. }\end{array}$ \\
\hline
\end{tabular}

Source : élaboration de l'auteur 


\section{Caractéristiques d'un district industriel a. La notion d'économies externes}

L'une des notions qui reviennent souvent lorsqu'on parle de district industriel Marshallien est celle des économies externes. Alfred Marshall se basa sur l'observation empirique de localités bien précises pour définir cette notion. Cette définition correspond globalement au concept d'économies d'agglomération de Weber (Weber, 1909). Il s'agit en effet pour Marshall des économies qui dépendent du développement général de l'industrie notamment :

- l'existence d'un marché de travail garantissant en permanence la disponibilité des compétences requises par les firmes ;

- le développement d'industries voisines dans la perspective de répondre aux besoins des firmes du district ;

- la stabilité de la demande qui, si elle diminue pour une industrie, sera compensée par la demande des industries connexes ;

- les économies provenant de la communication entre acheteurs et vendeurs.

Les économies décrites par Marshall au niveau du marché de travail sont reprises également dans les travaux de Weber sur « le développement de l'organisation du travail ». Ces économies proviennent des relations inter firmes et inter industries qui donnent lieu à l'externalisation de certaines activités de production. L'analyse de l'agglomération par Weber se croise également avec celle de Marshall au niveau des économies générées par la réduction des coûts fixes qui se répartissent entre les producteurs d'un même système local (Phelps,1992).

L'héritage laissé par Marshall et Weber est un concept plutôt éclectique d'économies externes/d'agglomérations qui consiste en une panoplie de facteurs pouvant potentiellement expliquer le phénomène de concentration spatiale de l'activité économique. Cette explication d'ordre général a deux caractéristiques majeures. D'abord, c'est une explication qui en cas d'utilisation inadéquate, peut réduire l'agglomération économique au simple principe de causalité cumulative. Ensuite, le concept d'économies externes/ d'agglomérations comprend une analyse limitée des raisons derrière l'externalisation des activités économiques par les firmes.

Marshall fit par ailleurs la distinction entre économies externes et internes. Il a ainsi défini les économies internes comme des économies d'échelles qui dépendent des ressources propres à la firme, contrairement aux économies externes qui dépendent du développementgénéral de l'industrie. Dans les travaux de Marshall, les économies internes sont présentées comme un processus dynamique cumulatif qui dépend fortement du management de la firme. Ceci suggère que ce processus a forcement une fin, compte tenu de 
l'espérance de vie de l'être humain et de la brièveté de la période de pleine jouissance de ses capacités mentales. Ceci amena Marshall à affirmer qu'une firme ne peut pas compter indéfiniment sur la qualité de son management au fil des générations. A l'inverse, les économies externes permettent à la firme de développer son activité tout en garantissant des coûts en baisse à une cadence constante. Etant connu par ses analogies biologiques, Marshall illustra cette idée de progrès et de déclin des firmes par la dynamique du milieu forestier dans lequel les arbres matures souffrant de perte de vitalité se trouvaient forcées à céder éventuellement leur place aux jeunes arbres en meilleure santé.

Dans le cadre de son analyse des économies externes, Marshall en identifia deux types : les économies de spécialisation qui proviennent des principes bien connus de l'efficience industrielle et des rendements croissants.Ces économies sont plutôt présentes dans les organisations industrielles opérant dans des contextes spécifiques, notamment un contexte technologique et légal dans lequel les différentes phases complémentaires du processus de production sont réalisées par plusieurs firmes spécialisées. Il y a également économies de spécialisation lorsque le jeu du marché requiert la différenciation et la variabilité en termes de quantité et de qualité.

Le second type d'économies externes est celui provenant de l'apprentissage et de la créativité. Elles se manifestent par le partage de compétences et de savoir-faire entre les firmes d'une même localité, et par l'apparition d'innovations technologiques et de solutions contractuelles. Cette montée en compétences, et cette floraison technologique résultent de l'interaction entre les différentes approches originales des firmes enracinées dans leur vie quotidienne, mais aussi des expériences de groupes de producteurs et de commerçants qui vivent et travaillent dans le district. La décentralisation de l'activité industrielle s'avère ainsi particulièrement intéressante quand elle renforce la capacité de groupes constitués d'employés des différentes firmes à communiquer et à innover de façon à différencier la production.

\section{b. L'aspect social}

Un district industriel ne peut être qualifié comme tel sans une communauté locale qui entretient un lien étroit avec le système de production. Il s'agit ici de relations dont une grande partie est de nature familiale, assurant ainsi un soutien moral mais aussi financier en cas de besoin. C'est ce groupe de personnes fédérées autour d'un système de valeurs solide définissant un compromis social, une éthique de travail, des règles d'échange et d'interaction, qui garantit au district sa flexibilité et par conséquent, son développement. Une caractéristique majeure de ce système est 
l'encouragement de l'entrepreneuriat et de l'innovation; une vision particulièrement adaptée au contexte du district et à ses besoins.

Cette vision est véhiculée à travers les différentes institutions qui interviennent dans le quotidien de la communauté comme l'école, l'église ou encore le marché. En effet, même les institutions à vocation politique participent à ce travail d' "homogénéisation de perception » sans que cela exclut la possibilité d'émergence de conflits d'intérêts entre elles et les membres du district. De telles situations sont normalement régies par égard pour l'intérêt suprême de l'ensemble de la communauté. G Becattini souligne un point qui n'est pas sans importance quant au fonctionnement du district. En effet, sa nature requérant un échange continu avec le monde extérieur, implique l'apparition d' " un flux migratoire » de personnes entrant et sortant de son périmètre, et donc un effort d'intégration sociale continue pour les nouveaux venus (Becattini, 1990).

\section{c. Les ressources humaines et le marché de travail}

Le marché de travail local d'un district industriel est caractérisé par la présence de deux grandes catégories de ressources humaines :

D'abord les ouvriers qui peuvent travailler à plein temps, à temps partiel ou depuis leurs domiciles et dont la mobilité est élevée du fait de la recherche perpétuelle du meilleur rapport « poste / aspirations ». En effet, le processus est continu dans le sens où un changement de poste ouvre naturellement les horizons sur de nouvelles opportunités qui n'étaient pas à la portée auparavant. Un «local pool of skills »est ainsi mis à la disposition des firmes leur épargnant l'effort et les coûts relatifs à la recherche de main d'œuvre qualifiée et leur conférant un avantage d'efficacité de recrutement par rapport aux grandes firmes qui peuvent être dotées de ressources plus importantes et de moyens plus sophistiqués. La compétence de l'ouvrier est ainsi considérée comme une sorte de bien public, propriété du district (Becattini, 1990).

Ensuite, il y a les entrepreneurs que l'on peut qualifier de « cerveaux » du district puisque c'est à eux qu'incombe la tâche d'étudier ce dernier d'un point de vue historique, social et culturel afin de dégager les produits qu'il est en mesure de fabriquer. Les entrepreneurs doivent être d'excellents prospecteurs attentifs aux besoins du marché et à l'écoute du consommateur qui n'est jamais très loin et qui développe un goût pour les produits « sur mesure ». Becattini donne une description détaillée qui dresse bien le profil de cet entrepreneur dans le cerveau du lecteur. Il le décrit comme quelqu'un qui ne possède pas sa propre usine employant plusieurs ouvriers, mais qui dispose d'un dépôt de matières premières qu'il achète lui-même et d'un nombre réduit de collaborateurs. Il travaille sur la conception de «projet de produit » sur la base des études de marchés préalablement réalisées ayant permis de détecter 
les tendances du marché. Ce projet composé d'une gamme d'articles est ensuite proposé à des producteurs du district afin d'évaluer la possibilité de le concrétiser à partir des matières premières disponibles en stock. Ce schéma, allant de la prospection du marché à la conviction d'un producteur d'adopter son projet, fait de l'entrepreneur avec le temps un expert du district.

Dans l'ouvrage collectif « industrial districts and interfirm cooperation in Italy », F.Pyke et W. Sengenberger reviennent sur les conditions de travail et de rémunération au sein d'un district, en énumérant les points de vue de différents chercheurs dans le domaine. Il convient dans un premier temps de rappeler que l'information se fait rare quant à ces aspects du fonctionnement du district industriel, et même quand elle existe, elle peut revêtir un caractère contradictoire. En effet, Trigilia soutient que le niveau de salaire dans un district est supérieur au standard défini sur le plan national du fait du phénomène de marchandage courant au niveau local. Ce point de vue est partagé par Brusco qui affirme que les salaires payés par les petites firmes d'un district industriel sont comparables en moyenne à ceux payés par les grandes firmes. Amin et Robins quant à eux sont plutôt sceptiques par rapport à cette vision optimiste et soutiennent que le développement de certains districts est étroitement lié au niveau bas des salaires.

S'agissant des conditions de travail, Brutti et Calistri les qualifient d'intolérables dans plusieurs cas, alors que Trigilia, Brusco, Lazerson et Becattini trouvent bien des raisons qui font du district un contexte de travail attrayant(Pyke et Sengenberger,1990). Il est possible de citer dans ce sens l'autonomie du travailleur et sa marge de manœuvre quant à la gestion du travail, la facilité de mobilité et le changement récurrent de statut qui varie entre employeur et employé, la valorisation des compétences qui est nettement meilleure par rapport au monde extérieur et la qualité de vie à l'extérieur du lieu de travail soit plus précisément l'environnement social: logement, activités culturelles, etc.

Cette diversité d'informations et de visions ne peut que témoigner de l'existence de différentes variantes du district industriel où les conditions de travail diffèrent. Elle confirme aussi l'absence d'un cadre théorique permettant l'évaluation des conditions de travail dans le contexte particulier du district. En effet, le cadre existant relatif à la production de masse ne peut contenir la spécificité du district vu la différence notable des deux modèles en matière de gestion de l'organisation du travail. Evaluer les conditions de travail dans un district est ainsi tributaire de la mise en place de moyens de mesure capables d'assimiler l'absence d'une ligne de démarcation entre vie professionnelle et vie personnelle, puisque le district est perçu comme une expérience sociale globale qu'il est difficile de décomposer ... c'est un mode vie. 


\section{d. Compétition et coopération}

Une caractéristique phare du district industriel est la coexistence de deux phénomènes économiques contradictoires que sont le coopération et la compétition; on parle alors de coopétition. La coopération est clairement illustrée par les liens de sous-traitance qu'implique l'organisation verticale du processus de production. La spécialisation des firmes fait que leurs produits et leurs prestations se complètent presque systématiquement. Il existe également un autre type de coopération que l'on qualifie d'horizontale consistant en des prestations de services « supports » comme le marketing, la comptabilité, le consulting ou d'autres services plus proches du cœur de métier, tel que le design dans le domaine de la mode. (Piore et Sabel, 1984)

Un niveau encore plus avancé de coopération est des fois atteint quand les entreprises cèdent les commandes qui dépassent leurs capacités de production à des concurrents potentiels. Cependant, ceci n'empêche l'existence d'une forte compétition entre les entreprises du district produisant des biens similaires. Trouver un équilibre entre la coopération et la compétition est essentiel à la survie du district puisque c'est ce qui lui permet de compenser les inconvénients inhérents à sa nature constituée d'entreprises de petite taille, et c'est cet équilibre qui lui confère son dynamisme et sa flexibilité.

Ce fut Alfred Marshall qui créa en premier le parallèle entre deux des plus grandes théories qu'a connu l'humanité, à savoir celle d'Adam Smith selon laquelle « dans chaque art, la division du travail, aussi loin qu'elle puisse être portée, donne lieu à un accroissement proportionnel dans la puissance productive du travail » (Smith,1986) et la théorie d'évolution de Darwin. Marshall présuma ainsi qu'en milieu économique comme dans n'importe quel milieu, le combat pour la survie privilégie le plus apte. Cette aptitude est liée à deux facteurs complémentaires que sont la différentiation et la capacité de coordination (Jong-II You et Frank Wilkinson, 1994)

En effet, afin de mieux cerner la notion de coopération, il convient dans un premier temps de la contextualiser par rapport à la division du travail. Selon Smith, la division du travail permet d'aiguiser les compétences des travailleurs grâce à la spécialisation, de réduire le temps passé dans le changement d'ouvrage et d'optimiser l'utilisation des ressources. Cette division de travail telle que décrite crée toutefois une dépendance entre les différentes étapes du processus de production d'où la nécessité de coopération entre les firmes qui cherchent chacune à garantir ses intérêts propres (Smith, 1986).

Si Smith n'a connu de l'industrialisation qu'un avant goût prometteur qui l'a poussé à réagir aux philosophies répandues de son époque notamment le mercantilisme, Marx a quant à lui connu cette ère. Le philosophe allemand a ainsi assisté à l'essor du capitalisme et de la division du travail, mais 
également aux crises cycliques et à la naissance de la classe ouvrière. Il fait la distinction entre la division sociale du travail que chaque société établit naturellement en fonction de critères tels que l'âge et le sexe, et la division technique ou manufacturière du travail qui s'est créée avec le capitalisme.

Marx fait par ailleurs la différence entre la coopération et la parcellisation (Marx, 1974). La première étant le simple fait que des ouvriers soient regroupés dans un même lieu afin de réunir leurs forces pour accomplir un travail, ce qui constitue un moyen d'accroitre la productivité sans devoir investir plus. S'agissant de la parcellisation, la connotation est nettement plus technique puisqu'elle consiste en la division d'un même processus de production en plusieurs tâches successives accomplies par des ouvriers spécialisés. Ces derniers se trouveront à terme isolés et inévitablement liés à leurs collègues accomplissant le reste des tâches. Ceci confère au capital une supériorité sur le travail résigné à lui vendre sa force. Ce qui a accentué la tendance à cette division manufacturière du travail, c'est le développement technologique et l'apparition de machines et de chaines de production ayant fait de cette coopération une nécessité.

Marshall consacra une bonne partie de son analyse à la notion de coopération qu'il plaça au-dessus même de la compétition constructive. Ainsi, selon lui, « même la compétition constructive est moins bénéfique que la coopération altruiste » (Marshall, 2009). La coopération peut toutefois revêtir un caractère « anti social » quand une catégorie d'acteurs tels que les unions ou les associations de commerce coopèrent contre les autres membres de la société afin de préserver leurs intérêts, ou encore comme les producteurs qui coopèrent contre un concurrent vendant un produit avec une marge bénéficiaire inférieure à ce qui leur convient. Il rajouta également que la compétition peut elle aussi être anti sociale, ce qui le fit conclure que le mot « compétition » n'est pas adapté pour décrire les caractéristiques spécifiques de la vie industrielle. Il propose donc de le remplacer par un terme n'impliquant pas de qualités morales mais qui indique le fait indiscutable que le monde moderne du business et de l'industrie est marqué par plus d'habitudes autonomes et de choix libres, ce terme serait: la liberté économique « economic freedom».

\section{e. Le progrès technologique}

Si dans une grande entreprise la résistance à l'introduction du progrès technologique est prononcée, c'est parce que cette décision provient d'une minorité managériale n'ayant pas consulté ses employés et n'ayant pas pris en considération leurs attentes et leurs opinions. Dans le district industriel, ce pas est perçu comme un processus social qui a lieu progressivement à travers la prise de conscience de l'ensemble des parties. Celles-ci partageant tous le principe de la primauté de l'intérêt collectif et la fierté du produit du district et 
de son processus de production. Elles voient ainsi en cette décision un pas vers un avenir meilleur même si elle peut impliquer des restructurations douloureuses (Becattini, 1990).

\section{f. Le système local de crédit}

Nul n'ignore la difficulté pour les petites entreprises d'accéder au financement vu leurs moyens limités et l'absence de garanties qu'elles peuvent présenter à l'organisme de crédit. Or le développement des firmes du district est tributaire d'un financement qu'elles ne peuvent se procurer en interne et que les organismes de financement externes sont peu disposés à leur apporter. Cette situation est remédiée par la théorie de « la banque locale ». Il s'agit d'une institution née et développée dans le district même, qui connait très bien l'ensemble de ses acteurs et spécialement les entrepreneurs et qui est impliquée dans la vie quotidienne de sa communauté.

Les caractéristiques de la banque locale font qu'elle a des critères d'octroi du financement bien différents de ceux de la banque classique : ils prennent en considération les qualités personnelles de la personne qui demande le crédit ainsi que les perspectives de l'investissement qu'elle compte faire (Becattini, 1990). Ceci n'empêche l'existence d'une vérification logique et raisonnable des conditions économiques d'octroi du crédit vu l'enjeu considérable sur l'ensemble des membres du district. En effet, la structure dense du district et la forte interdépendance entre ses acteurs peut être source de danger dans ce contexte dans la mesure où si un acteur se trouve en situation de difficulté, plusieurs autres dont le travail dépend du premier, risquent de suivre ; d'où la sensibilité de la décision de la banque locale.

\section{g. Le dynamisme et l'auto reproduction du district industriel}

Le district industriel est par sa nature dynamique et auto-reproductive. Ceci est principalement dû à la comparaison perpétuelle imposée par la rude concurrence externe, établie entre le coût de production en interne et celui de sous-traitance ou du fait de «faire faire » en externe. Il ne s'agit toutefois pas de comparer généralement le fait de produire soi même et d'acheter. On est dans une optique de coopération ou de « doing together ». La firme en question s'intéresse particulièrement à qui ou avec qui elle va prendre ce pas, comment, où et quand. Le point de comparaison invisible étant toujours ce qui se fait à l'extérieur du district et la manière dont il se fait.

Il faut noter que la notion de coût qui constitue la base de la comparaison antérieurement évoquée, est fortement imprégnée par la culture mêmedu district et son contexte social, ce qui la rend différente de la notion classique du coût. En effet, ce qui peut être considéré comme un coût pour la grande firme ne prenant pas en considération les aspects historiques, sociaux et culturels, peut ne pas l'être dans un district qui se définit avant tout comme 
une entité socio économique. La décision de produire soi même ou de faire faire en externe n'est donc jamais purement économique.

\section{Conclusion}

Le concept de district industriel a fait l'objet de plusieurs travaux de recherche, en particulier par les économistes italiens étant donné le succès sans précédent qu'il a connu dans leur pays. Cet article en constitue une présentation générale, il ne prétend cependant pas renseigner le lecteur sur tous les débats le concernant. Les lectures approfondies ayant permis la réalisation de cette revue de littérature dessinent une image globalement claire et cohérente du district industriel et de ses principales caractéristiques. Nous le définirions ainsi comme un système productif géographiquement délimité reposant sur une communauté de firmes (généralement petites) et de personnes qui y exploitent leur savoir faire, dans le cadre d'une relation d'appartenance et de bénéfice mutuel. Le paradigme de la spécialisation flexible est au cœur $\mathrm{du}$ fonctionnement du district et le processus de production y est divisé en plusieurs étapes, chacune accomplie par une firme. C'est à cette forme organisationnelle qu'est dû le climat particulier de coopétition (coopération et compétition) qui imprègne le district. Une coopération entre les firmes dont les tâches sont complémentaires et une compétition entre celles accomplissant les mêmes tâches.

Outre les variantes du district sur le terrain ayant animé le débat entre districtologues, la question de sa fiabilité comme mode de production a longuement été discutée, et ce en raison de sa présentation comme l'opposé du modèle de la production de masse. Entre ceux qui affirment que le district industriel a été salvateur pour certaines économies après la crise du fordisme, et ceux qui considèrent sa réussite comme le résultat exceptionnel de la réunion de plusieurs conditions spécifiques, nous pensons que le développement économique ne peut se résumer à une formule valable en toutes circonstances. Chaque pays (voire chaque région) est différent par son histoire, la structure de son économie, son organisation sociale, le savoir faire de sa population, etc. Il revient donc aux décideurs d'établir des politiques permettant d'accompagner le pays/ la région en tenant compte de ses spécificités, dans une perspective de capitalisation sur les acquis et de mise en valeur des ressources et du savoir faire. Le résultat peut prendre plusieurs formes, notamment des systèmes productifs locaux semblables aux districts, des grandes entreprises rappelant celles de l'ère fordiste ou encore des champions nationaux qui sont nés petits mais qui ont pu se développer pour devenir de grandes multinationales. Bien qu'il puisse globalement s'inscrire dans un modèle particulier, le développement économique reste une trajectoire propre que chaque pays doit dessiner par lui-même dépendamment de ses spécificités. 


\section{References:}

1. Amin, A. (1994). Post-Fordism: Models, Fantasies and Phantoms of transition. In Amin, A. (ed.), Post-Fordism: a reader (pp. 1-39). Oxford: Blackwell Publishers.

2. Becattini, G. (1990). The Marshallian Industrial district as a socioeconomic concept. In Pyke,F., Becattini, G. and Sengenberger,W. (eds), Industrial district and inter-firm cooperation in Italy (pp. 3750). Geneva, IILS.

3. Boyer, R. (1986). La théorie de la régulation: une analyse critique. Paris : La Découverte.

4. Freeman, C. and Perez, C. (1988). Structural crisis of adjustment, business cycles, and investment behavior. In G. Dossi, C. Freeman, R. Nelson, G. Silverberg and L. Soete. (Eds), Technical Change and Economic Theory (pp. 39-62). Pinter, London.

5. Hirst, P. and Zeitlen, J. (1989). Flexible specialisation and the competitive failure of UK manufacturing. Political Quarterly.

6. Hirst, P. and Zeitlen, J. (1991). Flexible specialization versus postfordism: theory, evidence, and policy implications. Economy and society.

7. Jessop, B. (1992 b). Post Fordism and flexible specialization: icommensurable, contradictory, complementary, or just plain different perspectives? In H. Ernste and V. Meier (eds), Regional development and contemporary industrial response: Extending flexible specialization. London: Belhaven.

8. Jong-II You and Frank Wilkinson. (1994). Competition and cooperation: toward understanding industrial districts. Review of Political Economy.

9. Marshall, A. (2009). Principles of Economics: Unabridged Eighth Edition.

10. Marx, K. (1974). Capital. London: Lawrence and Wishart.

11. Nielsen, K. (1991). Towards a flexible future-theories and politics. In B. Jessop, H. Kastendick, K. Nielsen and O. Pedersen (eds), The politics offlexibility. Aldershot: Edwaed Elgar.

12. Phelps, N. (1992). External economies, agglomeration and flexible accumulation. Transactions of the Institute of British Geographers, New Series, Vol. 17, No. 1.

13. Piore, M. and Sabel, C. (1984). The second industrial divide: Possibilities for Prosperity. New York: Basic Books.

14. Pyke, F. and Sengenberger, W. (1990). Introduction. In Pyke,F., Becattini, G. and Sengenberger,W. (eds), Industrial district and interfirm cooperation in Italy (pp. 1-9), Geneva, IILS 
15. Sabel, C. and Zeitlen, J. (1985). Historical alternatives to mass production: politics, markets and technology in nineteenth century industrialization. Past and Present.

16. Smith, A. 1986. Wealth of nations. London: Penguin Books.

17. Weber A. (1909), Theory of the Location of Industries. CSISS Classics.

18. Weber, M. (1971) Economie et société, traduction partielle de Weber (1922 b), Paris, Plon, nouvelle éd. 1995. 